\title{
A review of the safety of common aesthetic procedures during pregnancy
}

\begin{abstract}
The prevalence of aesthetic procedures has increased exponentially over the last decade. Pregnancy causes many aesthetic changes in a woman, such as melasma, hirsutism and striae. Although these are temporary in most cases, they can be distressing to the mother. As such, the desire to undergo aesthetic procedures can increase during this period. A variety of procedures exist, however, their safety profiles have usually been determined by tests on non-pregnant volunteers. The changes that occur in pregnancy and the presence of a foetus, which is susceptible to a wider range of toxins, means this data cannot be directly transferred to dictate pregnancy safety profiles. In general, most aesthetic procedures and agents are deemed to be safe to use in pregnancy, however, the health of both mother and foetus should be paramount. Aesthetic procedures are non-urgent, therefore, best practice would be to centre consultations on patient counselling, encouraging patients to delay procedures until after pregnancy.
\end{abstract}

Volume 2 Issue 5 - 2017

\author{
Jakharia-Shah $\mathrm{N},{ }^{\prime}$ Chadha $\mathrm{P}^{2}$ Watson $\mathrm{L}^{3}$ \\ 'King's College London, UK \\ ${ }^{2}$ St Thomas' Hospital, London, UK \\ ${ }^{3}$ King's College London, UK
}

Correspondence: Nihull Jakharia-Shah, 53 Sedgecombe Avenue, London, UK, Tel 07739397198,

Email nihullshah@hotmail.com

Received: June 21, 2017 | Published: August 18, 2017

Keywords: pregnancy, cosmetic procedures, safety profiles, best practice

\section{Introduction}

The prevalence of aesthetic procedures has increased exponentially over the last decade. The British Association of Aesthetic and Plastic Surgeons (BAAPS) stated that over 51,000 people in UK underwent a cosmetic procedure in 2015. ${ }^{1}$ This number has risen year-by-year and is forecast to continue growing in the future. For many women, being pregnant is not a barrier to receiving simple aesthetic procedures. ${ }^{2}$ The aesthetic changes that occur during pregnancy, such as melasma, hirsutism and striae, ${ }^{3}$ can be dramatic and cause significant upset to the mother, thus, pregnancy can increase, or initiate, a woman's desire to receive aesthetic treatment.

The delivery of any treatment during pregnancy, whether medical or aesthetic, is a contentious topic. The debate on the safety of these procedures centres on the transient, but significant, physiological changes that occur during pregnancy and the sharing of blood and nutrients (or toxins) between mother and foetus. Due to the risks and ethical considerations involved, drugs are rarely tested on pregnant women. Thus, data on safety profiles is limited in this patient group. The differences in physiology make it inappropriate to translate data from trials in non-pregnant women to dictate practice during pregnancy.

Due to unconvincing, or absent, safety information on aesthetic products practice differs greatly between professionals. The general consensus is to defer non-essential procedures until after pregnancy. ${ }^{4}$ However, it should also be noted that aesthetic changes can cause devastating effects to the mother's mental health, potentially disrupting her relationship with her child as well as her ability to care for the child. In such situations the advantages and disadvantages of the procedure must be evaluated to reach a decision which creates the best possible outcome for mother and child. In this article, we review the most up-to-date information on the safety profiles of the most commonly requested aesthetic procedures during pregnancy and have outlined a framework for the general management of patients seeking these procedures.

\section{Discussion}

\section{General procedural risks during pregnancy}

A significant risk to consider from any procedure which causes a break in the skin is infection. This is especially important in pregnancy as infections can lead to maternal and foetal complications such as, sepsis, chorioamnionitis, intra-uterine growth restriction, malformations and prematurity. Increased precaution is therefore needed to prevent infection, including hand washing, gloving and appropriate skin preparation. Iodine and Hexachlorophene are both contraindicated in pregnancy due to thyroid and central nervous system toxicity, respectively. ${ }^{5}$ Therefore, alcohol or Chlorhexidine gluconate solutions should be used to achieve sterility of the procedure site.

Alongside the increased risk of infection there is also a difficulty in treating infections during pregnancy as all common antibiotics are classified as either Category B or C by the old FDA guidelines. ${ }^{6}$ The category based system was replaced by the FDA in 2015, however, since most reviews pre-date this we will discuss products in terms of this system. A breakdown of the classification system is shown below:

Category $\quad \begin{aligned} & \text { Definition } \\ & \text { Well-controlled studies in pregnant women } \\ & \text { have failed to demonstrate a risk to the foetus } \\ & \text { at any stage in pregnancy }\end{aligned}$
$\begin{aligned} & \text { Animal studies have failed to demonstrate a risk } \\ & \text { to the foetus but there are no well-controlled } \\ & \text { studies in pregnant women }\end{aligned}$
$\begin{aligned} & \text { Animal studies have shown an adverse effect } \\ & \text { on the foetus and there are no well-controlled } \\ & \text { studies in humans, but potential benefits may } \\ & \text { warrant the use of the drug in pregnant women } \\ & \text { despite potential risks }\end{aligned}$


Table Continued.

\section{Category Definition}

There is positive evidence of human foetal risk based on adverse reaction data from investigational or marketing experience or studies in humans, but potential benefits may warrant use of the drug in pregnant women despite potential risks

Studies in animals or humans have X demonstrated foetal abnormalities, and the risk involved in using the drug in pregnant women clearly outweigh any potential benefits

The risk of significant bleeding from a procedure should be also considered prior to performing procedures during pregnancy. Pregnant women are more susceptible to anaemia and it is linked with adverse outcomes for both mother and foetus in the event of major haemorrhage. However, pregnancy itself is a hypercoagulative state so the risk of developing a significant bleed during simple aesthetic procedures is reduced.

Wound healing during pregnancy does not pose a significant health threat, but creates an argument against the delivery of aesthetic procedures. Due to changes in hormones and tension factors, pregnancy is linked with the development of hypertrophic and keloid scarring. ${ }^{8}$ There is also an increased incidence of hyperpigmentation during pregnancy. ${ }^{9}$ Therefore, performing aesthetic procedures during pregnancy could lead to worse aesthetic outcomes in the long-term. This should be discussed with the patient during the consenting process prior to any procedure.

\section{Chemical peels}

A variety of chemical peels exist in the market and are used for multiple purposes, including the treatment of melasma. However, studies proving its use in the treatment of melasma excluded pregnant women from the trials. ${ }^{10} 2 \%$ Lactic acid is known to be used to treat gestational acne without any reported negative pregnancy outcomes. ${ }^{11}$ The product has a low level of dermal penetration, meaning negligible systemic effects, and could therefore be considered safe for use during pregnancy.

Lactic acid is also used in conjunction with salicylic acid and resorcinol in a combination known as a Jessner peel. This is a medium-depth peel, meaning deeper dermal penetration, which makes its use more contentious especially due to the inclusion of salicylic acid. It produces up to $25 \%$ dermal penetration, however this would likely result in a negligible systemic concentration. Salicylic acid is classified as a Category $\mathrm{C}$ drug for pregnancy according to the aforementioned FDA guidelines, thus, its use during pregnancy is acceptable depending on specific patient factors. Studies on the effects of cutaneous salicylic acid in pregnancy are limited, both on its own and as part of a Jessner peel. However, many large studies have evaluated the safety of low-dose oral acetylsalicylic acid (Aspirin) in pregnancy. This is commonly used as a prophylactic agent in women who are at a high risk of developing hypertension and pre-eclampsia. ${ }^{12}$ The studies concluded that there was no increase in the baseline rate of adverse events such as preterm birth, major malformations or low birth weights. ${ }^{13}$ Since the absorption from topical application would produce even lower systemic concentrations it would be unlikely that these peels would cause adverse outcomes if used during pregnancy. However, it is still recommended that they are used with caution and coverage should be limited, and without occlusion, if used during pregnancy. ${ }^{14}$

Another popular chemical peel agent is glycolic acid, a product which is also widely used for acne treatment. Multiple studies on animals have shown adverse reproductive effects, however doses were significantly higher than those used in cosmetic products. ${ }^{15}$ No trials in pregnant women have been conducted. Glycolic acid could therefore be considered as a Category $\mathrm{C}$ product, meaning carefully considered use in pregnancy. The minimal dermal invasion means the product should be relatively safe to use in pregnancy as a topical peel. ${ }^{16}$

\section{Minor procedures}

Common minor procedures, including removal of benign lesions using cryotherapy, shaving, snipping, punch or shave removal and electrocautery, all have verified safety records in pregnant women. ${ }^{17}$

\section{Injectable anaesthetic agents}

Lidocaine is the most commonly used injectable local anaesthetic agent, it is used in the delivery of Botulinum toxin and dermal fillers. Lidocaine is classified as a Category B drug in pregnancy. Once in the blood stream it can cross the placenta and enter the foetal circulation. A study with nearly 300 pregnant women showed no relative increase in the number of adverse foetal events in women who had lidocaine administered during the first trimester of pregnancy. ${ }^{18}$ A more recent study analysing adverse pregnancy events in 210 women receiving local anaesthetic for dental procedures showed a slight but insignificant increase in adverse foetal outcomes compared to a control population $(4.8 \%$ versus $3.3 \%) .{ }^{19}$ Studies in rats have also failed to show an increase in adverse events following the use of lidocaine in pregnancy. ${ }^{20}$

The major risks from using lidocaine come from high-dose exposure or accidental arterial injection, both of which could theoretically lead to foetal cardiac or central nervous system toxicity. ${ }^{5}$ However, the maximum safe injectable dose of lidocaine is $4.5 \mathrm{mg} / \mathrm{kg}$, up to a maximum of $300 \mathrm{mg}$, which is well below the typical amount used for aesthetic procedures. High-dose or accidental arterial exposure can result in lidocaine toxicity in the mother, causing symptoms such as light-headedness, tachycardia, headaches and agitation. ${ }^{21}$ Once symptoms are present in the mother it can be assumed that the dose is significant to cause some level of foetal toxicity. It is reported that the concurrent use of a vasoconstrictor, such as adrenaline, alongside lidocaine can reduce toxicity by restricting systemic spread. Although this increases the safety of local anaesthetic, it also increases the risk profile as systemic absorption of adrenaline, such as through accidental intra-arterial injection, could lead to uterine artery spasm resulting in foetal compromise. ${ }^{22}$ However, these effects are minimised by the relatively small doses of adrenaline used in aesthetic injections; as low as 1 in 200,000 .

Other injectable local anaesthetics, such as Bupivacaine and Mepivacaine, are classified as Category $\mathrm{C}$ drugs for pregnancy and should therefore be avoided as there are safer alternatives. The use of Mepivacaine ${ }^{23}$ has been reported to cause complications such as foetal bradycardia, preterm labour and a doubling of congenital abnormalities. 


\section{Topical anaesthetic agents}

Lidocaine is also a popular topical anaesthetic agent but is usually combined with prilocaine when used in this form. The mixture is classified as Category B in pregnancy but increased risks are present due to the inclusion of prilocaine which has shown to produce methemoglobinaemia in the foetus when used during pregnancy. ${ }^{24}$

Benzocaine and tetracaine are other topical anaesthetic agents but both are Category $\mathrm{C}$ drugs due to more dermal penetration and a stronger association with foetal methemoglobinaemia. ${ }^{25}$ A lidocaine/ prilocaine mixture is therefore the preferred choice of topical local anaesthetic if required. An important note about topical anaesthetic agents is that their effectiveness increases with occlusion, however, penetration into the systemic circulation also increases with occlusion. Therefore, if used during pregnancy, occlusion should be avoided to minimise the risks of adverse events.

\section{Botulinum toxin}

Botulinum toxin is one of the most common aesthetic agents administered; however it also has various medical uses such as in achalasia and hyperhidrosis. Botulinum toxin is officially a Category $\mathrm{C}$ drug in pregnancy and manufacturer recommendations list pregnancy is a contraindication for its use. Very few trials of aesthetic administration of Botulinum toxin in pregnancy have been conducted, all suggesting a negligible increase in adverse foetal events. ${ }^{23}$ However, multiple trials have been conducted which analyse the effects of using for medical indications during pregnancy, including achalasia, migraine prophylaxis and cervical dystonia. Despite using higher concentrations of Botulinum toxin for medical purposes there is still a unanimous conclusion that there is no significant increase in adverse foetal outcomes compared to the general population. ${ }^{11}$

If administered correctly, intra-muscularly or intra-dermally, Botulinum toxin is unlikely to enter the systemic circulation. ${ }^{26}$ The large size of the molecule makes it unlikely to be able to cross the placental barrier. ${ }^{27}$ The doses of Botulinum toxin used for aesthetic procedures is also much lower than the estimated hazardous dose (100Units versus 600Units). ${ }^{27}$ The arguments for the safety of Botulinum toxin in pregnancy are convincing, however, it is believed that there is still insufficient data to confirm absolute safety of Botulinum toxin in pregnancy. Therefore, its use for medical conditions should be limited and undertaken with caution, and for aesthetic procedures, we would recommend that it is avoided.

\section{Dermal fillers}

There are numerous filler agents available on the market; the most common are collagen, hyaluronic acid, hydroxylapatite and poly-L-lactic acid. To our knowledge, there are no published trials assessing the safety of any filler agents during pregnancy. In the general population, the commonly reported complications of fillers consist of hypersensitivity reactions and injection site infections. ${ }^{28}$ In reality, fillers are probably safe for use in pregnancy, however, the lack of safety evidence and manufacturer recommendations stating that they should not be used during pregnancy mean their use should be delayed until after pregnancy if possible. Other factors to consider when administering fillers in pregnant women are the altered scar healing processes in pregnancy, susceptibility to hyperpigmentation and the concurrent use of lidocaine for the procedure. ${ }^{9}$

\section{Lasers}

There are a variety of lasers that can be used for aesthetic procedures; none have been tested for their safety profiles for aesthetic procedures during pregnancy. However, there are numerous studies evaluating their safety for medical treatments during pregnancy. Carbon dioxide lasers, ${ }^{29}$ neodymium-doped yttrium aluminium, ${ }^{30}$ holmium yttrium aluminium $^{31}$ and pulsed dye lasers ${ }^{32}$ have all been proven to be safe for use during pregnancy, however, the lack of data specifically relating to aesthetic procedures means it is generally advised to avoid using them until after pregnancy. There is a high risk of hyperpigmentation following the use of lasers, which would be accentuated during pregnancy, thus the long-term aesthetic outcome of laser treatment should be carefully considered before administering it.

\section{Conclusion}

Pregnancy is a time of great change for a woman, there are a plethora of physiological changes that occur, some of which manifest superficially in the form of melasma, hirsutism and striae, and, although temporary in most cases, these can be distressing to the mother. As a result she may seek specialist help to undergo procedures to counteract these changes. The scope of these procedures is vast, encompassing minor surgical procedures, chemical topical agents, lasers and injectable products such as Botulinum toxin or dermal fillers. These procedures have usually been tested and analysed on non-pregnant volunteers to determine their safety profiles, however, the changes that occur in pregnancy and the presence of a foetus, which is susceptible to a wider range of toxins, means this data cannot be directly transferred to dictate pregnancy safety profiles.

Minor procedures, including excision, cryotherapy and shave removal are all safe to perform during pregnancy. Chemical peels are generally considered safe; however, excessive systemic infiltration has been shown to cause adverse events in both animal and human studies. When considering more invasive procedures a secondary factor to consider is the use of anaesthetic agents, the most common of which is lidocaine. This has been shown to be the safest of the injectable and topical anaesthetic agents to use during pregnancy but it can produce adverse events in extreme circumstances. Injectable procedures, such as Botulinum toxin and dermal fillers, have limited studies evaluating their use for aesthetic purposes during pregnancy. They are assumed to be safe but manufacturer recommendations list pregnancy as a contraindication to their use.

In general, most aesthetic procedures and agents are deemed to be safe to use in pregnancy, however, in pregnancy the health of mother and foetus should be paramount. Aesthetic procedures are non-urgent and limited data on safety profiles, alongside studies which have shown even small risks adverse reactions, mean the benefits of having these procedures may not justify the risks involved. An important point to consider is the altered wound healing physiology during pregnancy as this can cause worse aesthetic outcomes for the patient. Best practice would be to centre consultations on patient counselling, reassuring them of the transient nature of many changes during pregnancy and of the short window of pregnancy during which they would be best to avoid treatments, encouraging patients to delay procedures until after pregnancy.

\section{Acknowledgements}

\section{None.}

\section{Conflict of interest}

There are no conflicts of interests for any of the authors in producing this article. 


\section{References}

1. The Private Clinic of Harley Street. How 2015 was the year for Cosmetic Surgery in the UK. 2016.

2. Marie C, Cabut S, Vendittelli F, et al. Changes in Cosmetics Use during Pregnancy and Risk Perception by Women. Int J Environ Res Public Health. 2016;13(4):383.

3. Afshar Y, Esakoff TF. The skin and pregnancy: Physiological changes and dermatoses. Contamporary OB/GYN. 2014.

4. Talakoub L, Wesley N. Cosmetic procedures in pregnancy. Dermatology News. 2015.

5. Richards KA, Stasko T. Dermatologic surgery and the pregnant patient. Dermatologic Surgery. 2002;28:248-256.

6. Lee KC, Korgavkar K, Dufresne RG, et al. Safety of Cosmetic Dermatologic Procedures During Pregnancy. Dermatologic Surgery. 2013;39:1573-1586.

7. Author unknown. FDA Pregnancy Categories. Drugs. 2016.

8. Gauglitz GG, Korting HC, Pavicic T, et al. Hypertrophic Scarring and Keloids: Pathomechanisms and Current and Emerging Treatment Strategies. Molecular Medicine. 2011;17(1-2):113-125.

9. Goldberg D, Maloney M. Dermatologic surgery and cosmetic procedures during pregnancy and the post-partum period. Dermatologic Therapy. 2013;26(4):321-330.

10. Sharquie KE, Al-Tikreety MM, Al-Mashhadani SA. Lactic Acid as a New Therapeutic Peeling Agent in Melasma. Dermatologic Surgery. 2005;31(2):149-154.

11. Trivedi MK, Kroumpouzos G, Murase JE. A review of the safety of cosmetic procedures during pregnancy and lactation. Int $J$ Womens Dermatol. 2017;3(1):6-10.

12. Allen KM, Green A, Wallace SVF. Use of low-dose aspirin in pregnancy-how will the nice 'hypertension in pregnancy' guideline alter current practice? ADC Fetal \& Neonatal Edition. 2011;96:112-113.

13. James AH, Brancazio LR, Price T. Aspirin and reproductive outcomes. Obstet Gynecol Surv. 2008;63(1):49-57.

14. Bozzo P, Chua-Gocheco A, Einarson A. Safety of skin care products during pregnancy. Can Fam Physician. 2011;57(6):665-667.

15. Munley SM, Kennedy GL, Hurtt ME. Developmental toxicity study of glycolic acid in rats. Drug Chem Toxicol. 1999;22(4):569-582.

16. Andersen FA. Final report on the safety assessment of glycolic acid, ammonium, calcium, potassium, and sodium glycolates, methyl, ethyl, propyl, and butyl glycolates, and lactic acid, ammonium, calcium, potassium, sodium, and TEA-lactates, methyl, ethyl, isopropyl, and butyl lactates, and lauryl, myristyl, and cetyl lactates. Int J Toxicol. 1998;17(1):1-241.
17. Meyer E. When the Patient is Pregnant. The Dermatologist. 2010;18(5).

18. Heinonen Op, Sloane D, Shapiro S. Birth defects and drugs in pregnancy. Littleton. 1977:357-365.

19. Hagai A, Diav-Citrin O, Shechtman S, et al. Pregnancy outcome after in utero exposure to local anesthetics as part of dental treatment: A prospective comparative cohort study. J Am Dent Assoc. 2015;146(8):572-580.

20. Fujinaga M. Assessment of teratogenic effects of lidocaine in rat embryos cultured in vitro. Anesthesiology. 1998;89(6):1553-1558.

21. Sweeney SM, Maloney ME. Pregnancy and dermatologic surgery. Dermatol Clin. 2006;24(2):205-214.

22. Fayans EP, Stuart HR, Carsten D, et al. Local anesthetic use in the pregnant and postpartum patient. Dent Clin North Am 2010;54(4):697-713.

23. Moore PA. Selecting drugs for the pregnant dental patient. J Am Dent Assoc. 1998;129(9):1281-1286.

24. Chen BK, Eichenfield LF. Pediatric anesthesia in dermatologic surgery: when hand-holding is not enough. Dermatol Surg. 2001;27(12):1010-1018.

25. Guay J. Methemoglobinemia related to local anesthetics: A summary of 242 episodes. Anesth Analg. 2009;108(3):837-845.

26. Tan M, Kim E, Koren G, Bozzo P. Botulinum toxin type A in pregnancy. Can Fam Physician. 2013;59(11):1183-1184.

27. Morgan JC, Iyer SS, Moser ET, et al. Botulinum toxin A during pregnancy: a survey of treating physicians. J Neurol Neurosurg Psychiatry. 2006;77(1):117-119.

28. Lolis M, Dunbar SW, Goldberg DJ, et al. Patient safety in procedural dermatology: Part II. Safety related to cosmetic procedures. J Am Acad Dermatol. 2015;73(1):15-24.

29. Gay C, Terzibachian JJ, Gabelle C, et al. Carbon dioxide laser vaporization of genital condyloma in pregnancy. Gynecol Obstet Fertil. 2003;31(3):214-219.

30. Buzalov S, Khristakieva E. The treatment of neglected cases of condylomata acuminata in pregnant women with the Nd: Yag laser. Akush Ginekol (Sofiia). 1995;34(2):38-39.

31. Adanur S, Ziypak T, Bedir F, et al. Ureteroscopy and holmium laser lithotripsy: Is this procedure safe in pregnant women with ureteral stones at different locations? Arch Ital Urol Androl. 2014;86(2):86-89.

32. Carlan SJ, Schorr SJ, Ebenger MF, et al. Laser lithotripsy in pregnancy. A case report. J Reprod Med. 1995;40(1):74-76. 